\title{
Rapid Progress and Limitations of Observational Astronomy in Japan
}

\author{
Norio Kaifu \\ NAOJ, and Open University of Japan 2-11 Wakaba, Mihama-ku, Chiba, 261-8586, Japan \\ E-mail:kaifunorio@aol.com
}

Observational astronomy and instrumentation in Japan remained weak until the 1970's, and the size of the astronomical community was quite small in spite of activities in theoretical astronomy/astrophysics. Big jumps towards the frontier of astrophysical observations with forefront telescopes were made during the1970's and 1980's in the fields of mm-wave and X-ray astronomy. Rapid progress followed in optical/IR astronomy. We report here the brief history, motivation, and ruling factors of such jumps in Japanese astronomy as well as their limitations. We also discuss the importance of building systematic regional cooperation.

Keywords: development of astronomy, large telescopes, national observatory, regional cooperation.

Accelerating the Rate of Astronomical Discovery - sps 5

International Astronomical Union, XXVII General Assembly

Rio de Janeiro, Brazil

August 11-14 2009 


\section{Introduction and Background}

Under the unique title of this IAU GA special session 5, I present my paper on two aspects of "acceleration". One is, of course, more and more discoveries in science. The second aspect of acceleration is to increase the number of players in astronomical discoveries, and to share the excitement of discovery among as many nations as possible. The IAU proposed the Strategic Plan "Astronomy for the Developing World" in the XXVII ${ }^{\text {th }}$ GA as a natural extension of the IYA 2009 which had involved 147 nations and had been very successful. The proposal of the Strategic Plan states that "The long term vision of the plan is that eventually all countries should participate at some level in astronomical research and that all children throughout the world will be exposed to knowledge about astronomy and the Universe". The case of Japan is only one of many stories but it certainly added a new nation and people outside the "western world" to the community of active astronomical research. We see many more nations and regions are now coming into the wonderland of astronomy, and that trend should be "accelerated".

Now let me give a brief view of Japanese astronomy in 1960's and 1970's as a background to this talk. Japan introduced modern science and technology late in the 19th century as an immediate result of Meiji Restoration. Japan joined the IAU in 1920, in the second year of its establishment, and played some positive roles (Shin Hirayama of University of Tokyo served as a vice president of IAU from 1922 to 1928, for example), but the scientific activities were mostly in theory. After World War II, M.Oda, T.Takakura, T.Hatanaka, N.Wakai and H.Tanaka started solar radio astronomy (1949) and they were among the earliest radio astronomy groups in the world (see Figure 1). A solar corona observatory was also established on the Mt. Norikura (1950) but was not very successful due to weather conditions. The 88 inch optical telescope at Okayama constructed by Grubb \& Parsons (1960) was the first "large telescope" and it prepared the base of Japanese astrophysical observations. At the beginning of the 1960's Japan was still a "developing country" in astronomy; only 3 Universities had a division of astronomy, and the number of Japanese IAU members was 18.

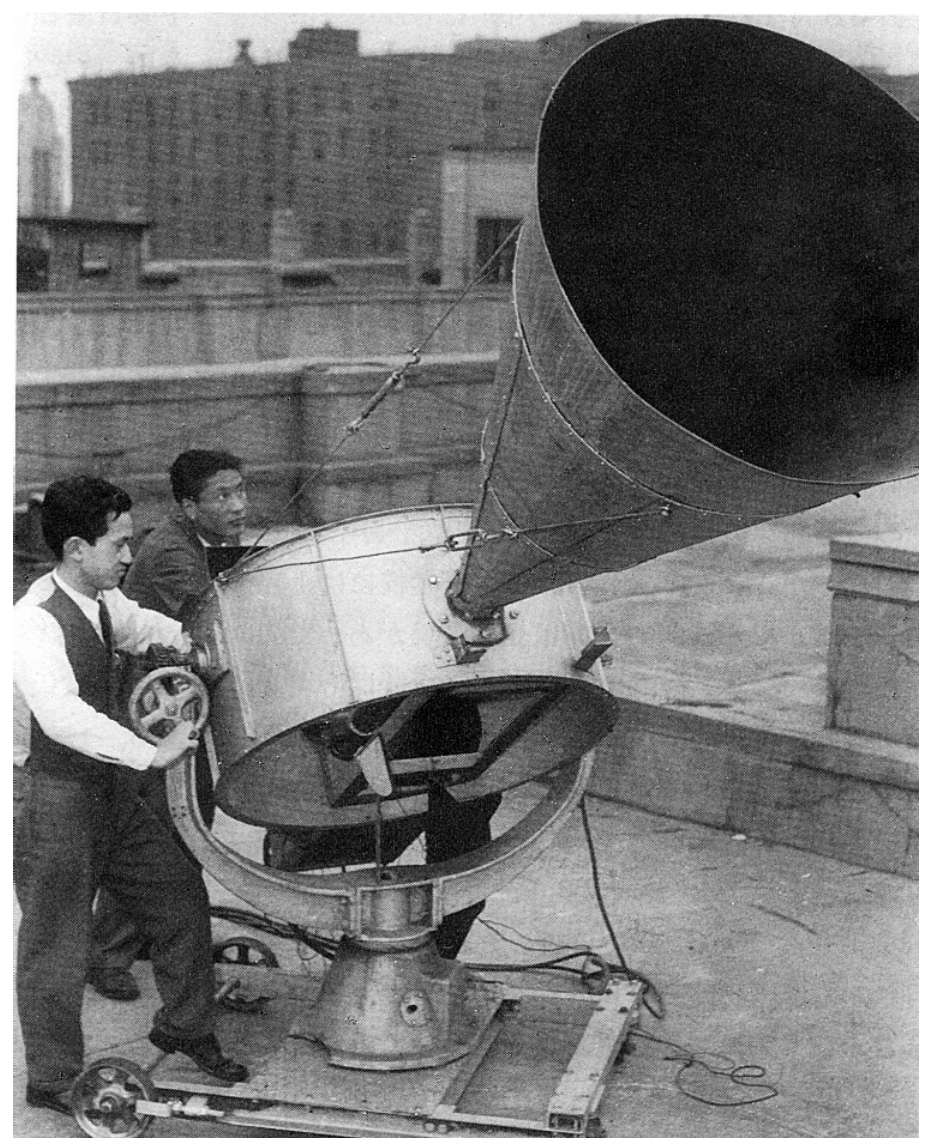

Figure 1. A historical photo. Minoru Oda (left) and Tatsuo Takakura are observing solar radio emission with their hand-made antenna (Ohsaka City University, 1950). 
Big jumps in the mm-wave and in the X-ray started in the 1970's and bloomed in the 1980's. The Radio astronomy group of Tokyo Astronomical Observatory (TAO) of University of Tokyo, K.Akahabe, M.Morimoto, N.Kaifu et al. constructed a $6 \mathrm{~m}$ aperture mm-wave telescope at Mitaka as the first cosmic radio telescope in Japan and started search and observations of interstellar molecular lines in the mm-wavelength region (1970). The $6 \mathrm{~m}$ telescope was followed by the detailed plan of a very large $45 \mathrm{~m}$ diameter $\mathrm{mm}$-wave telescope, aiming the establishment of radio spectroscopy based on interstellar molecular lines.

In space science, Japan launched the first satellite "Shinsei" in 1971 by $\eta$-rocket developed by H.Itokawa et al. (ISAS, U-Tokyo). Then the successful launches of "Taiyo" (1975) and "Hakucho" (1979) initiated the series of solar and cosmic X-ray satellites (by M.Oda, S.Hayakawa, Y.Tanaka, K. Tanaka et al.). Currently, Japanese space astronomy is very well known by its high activities; a 50-cm aperture Solar telescope Hinode, an X-ray telescope Suzaku, and a $70 \mathrm{~cm}$ aperture cooled IR telescope Akari are in operation. In the next section I discuss ground-based astronomy developments mainly because the developments of ground- based and space-borne astronomy has been running almost in parallel. I will come back to the space astronomy in the $3 \mathrm{rd}$ section.

\section{Jumps of Japanese Ground-Based Astronomy}

The Development of Japanese ground-based astronomy had expected two steps of a big jump; the first was in radio astronomy in early 1980's (from Mitaka 6-m to the Nobeyama 45-m mm-wave telescope), and the second was in optical/IR astronomy in late 1990's (from Okayama 1.88-m to the 8.2-m Subaru telescope). The basic plan of the Nobeyama $45-\mathrm{m} \mathrm{mm}$-wave telescope was settled in 1968 , fortunately at the same time as J. Welch and C. Townes discovered the spectra of NH3 and H2O, the first multi-atom molecules in interstellar region. We set the main target of the $45 \mathrm{~m}$ telescope, the world largest mm-wave telescope when realized, on the establishment of radio spectroscopy based on interstellar molecular lines, because a tremendous number of rotational spectra of small molecules were known to exist in the mm-wavelength region. Radio astronomy had been poor in spectral lines, and we expected that mm-wave spectroscopy would reveal the low temperature part of interstellar matter and would be a new powerful tool for observations of star formation, structure $\&$ dynamics of galaxies, and evolution of interstellar matter. The mm-wave spectroscopy was a wonderful gateway to new discoveries and to join the community of radio astronomy which had already been well established in the longer wavelength region with the 40 to $60 \mathrm{~m}$ class giant telescopes in USA, Europe and Australia.

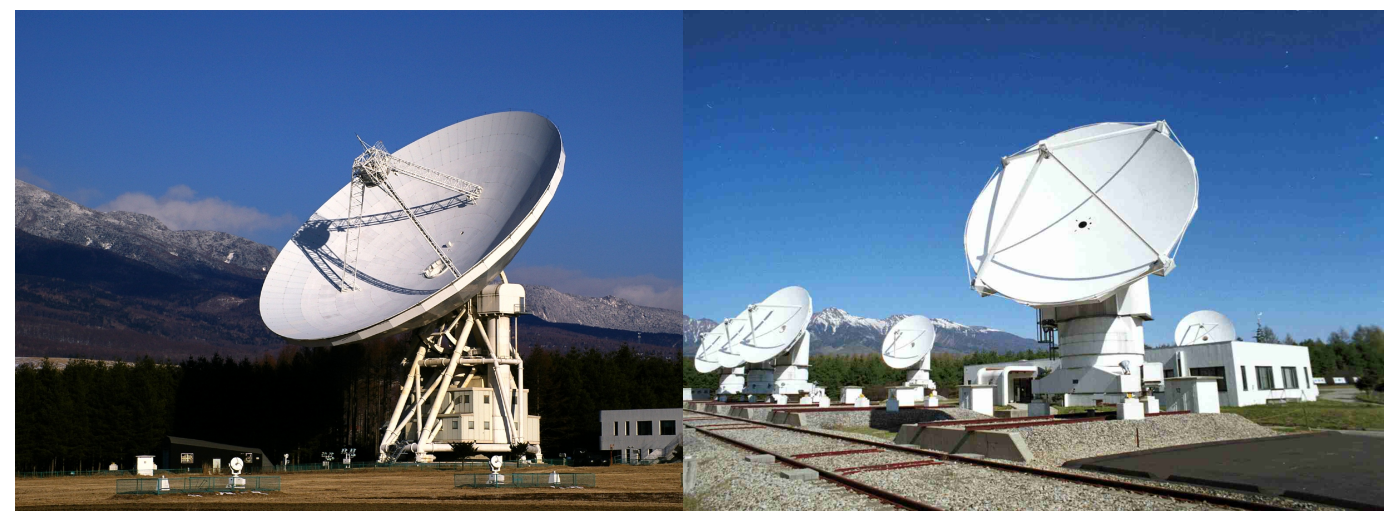

Figure 2. Nobeyama mm-wave telescopes: 45-m telescope (left) and 5-element interferometer (NAOJ).

To accomplish our target we developed new technologies, cooperating with engineers of several industries to achieve very high surface accuracy of the $45 \mathrm{~m}$ dish; the homologous deformation design, the automatic surface measuring laser instrument, carbon-fiber reflection panels, uniform temperature control of the dish structure etc. Also we developed a new type of very large $(32,000$ channel) and stable radio-spectrometer by applying the acousto-optical technology to match the observation capability of the $45 \mathrm{~m}$ telescope. The construction started at Nobeyama highland in 1978 and was completed in 1982. The five element mm-wave interferometer was completed two years later (M. Ishiguro et al.). We took almost 15 years to realize the $45 \mathrm{~m} \mathrm{~mm}$-wave telescope but it still kept its advantages. The Nobeyama $45 \mathrm{~m}$ telescope and the Interferometer contributed to make many exciting discoveries, such as the detection and identification of numbers of new interstellar organic molecules in 
dark clouds (by N. Kaifu, H. Suzuki, M,Ohishi et al.), detailed observations of bipolar outflows, protostellar cores and proto-planetary disks (by T. Hasegawa, R, Kawabe, M. Hayashi, S, Hayashi et al.), first observational confirmation of super-massive black hole (in NGC4268, by N. Nakai, M. Miyoshi et al.),etc. Many young astronomers had grown at Nobeyama, and excellent international cooperation like the Japan-UK mm-IR cooperation had been successfully made. The one-third of Nobeyama users were from abroad.The Atacama Large mm Array (ALMA), under construction on the Atacama Plateau in Chile by a collaboration of North America, Europe and Japan+Taiwan, is a challenging and natural extension of Nobeyama.

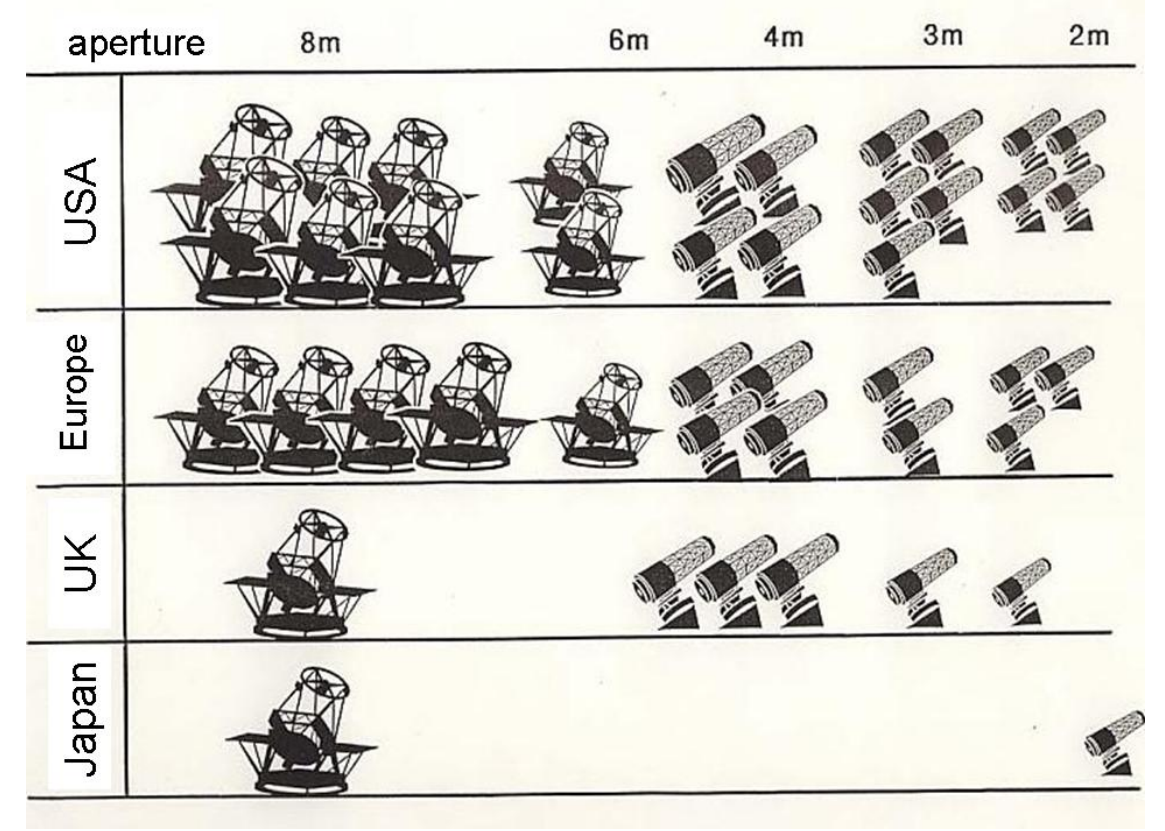

Figure 3. A figure which I used for the Subaru project explanation (around 1990), showing the big gap we had to jump over. The two types of telescope show the traditional and new generation designs.

The Second Jump of Japanese ground-based astronomy was the SUBARU Telescope construction, during 1991-2000. Figure 3 is one of my promotion figures which I used to explain the situation of Japanese (optical) astronomy, and shows what I meant "big Jump" in Japanese astronomy. In the time of preparation of the Subaru telescope (around 1990) the "Western World" already had many 3 to $4 \mathrm{~m}$ class telescopes and been testing new technology telescopes, thus were proceeding to new generation telescopes of 8-10 m class step by step. Japan, instead, had only $1.88 \mathrm{~m}$ old-fashioned telescope bought from UK in 1960 (see the big gap in figure 3). We convinced people that we had to jump this gap, as we had in radio astronomy from the $6 \mathrm{~m}$ to the $45 \mathrm{~m} \mathrm{~mm}$-wave telescope, otherwise we might never be able to touch the front-cut discoveries in the optical/IR astronomy. Actually there was a hot and endless discussion in the Japanese community of optical/IR astronomy for many years about the way to go, to challenge the world top or to realize a telescope with reasonable size with shorter time. The final decision was made by the committee for astronomy of the Science Council of Japan. Thanks for excellent leaders! The figure 1 was also a caution to ourselves against our optimism during this very challenging project.

In the early 1990's at least seven 8 m class telescopes with similar concepts were being prepared in parallel, so again Japanese astronomy, as a newcomer in the optica/IR astronomy world, needed good gateways to steer the Subaru Telescope towards new discoveries. For that purpose we first decided to work on the telescope and dome design to get as best an image quality as possible. We also planed a variety of observing instruments, among them were the Suprime-Cam for observational cosmology and the CIAO for exo-solar planets. The construction of the Subaru Telescope was 19912000 and we were fortunate again! The discovery of exo-solar planets by Mayor et al occurred in 1995, and the discovery of dark energy was in the later half of 1990's. The CIAO, Coronagraphic Imager with Adaptive Optics, was proposed in 1992 to get direct images of exo- solar planets and protoplanetary disks around newly-born stars. The star and planet formation has been a strong field in Japan 
for many years and CIAO produced good front-cutting data especially of disks, and now the 2nd generation CIAO (High-CIAO) with ten-times higher sensitivity than the first generation has started test observations. The Suprime-Cam on the prime focus of Subaru Telescope has also been a unique instrument among the 8-10 m class telescopes. Its production late of cosmological observations has been very high since its operation including the detection of furthermost galaxies, imaging of dark matter cooperating with HST and other telescopes, discoveries of various new phenomena in distant universe, etc. The 2nd generation Suprime-Cam (Hyper Suprime-Cam) with ten-times larger FOV is also under construction.

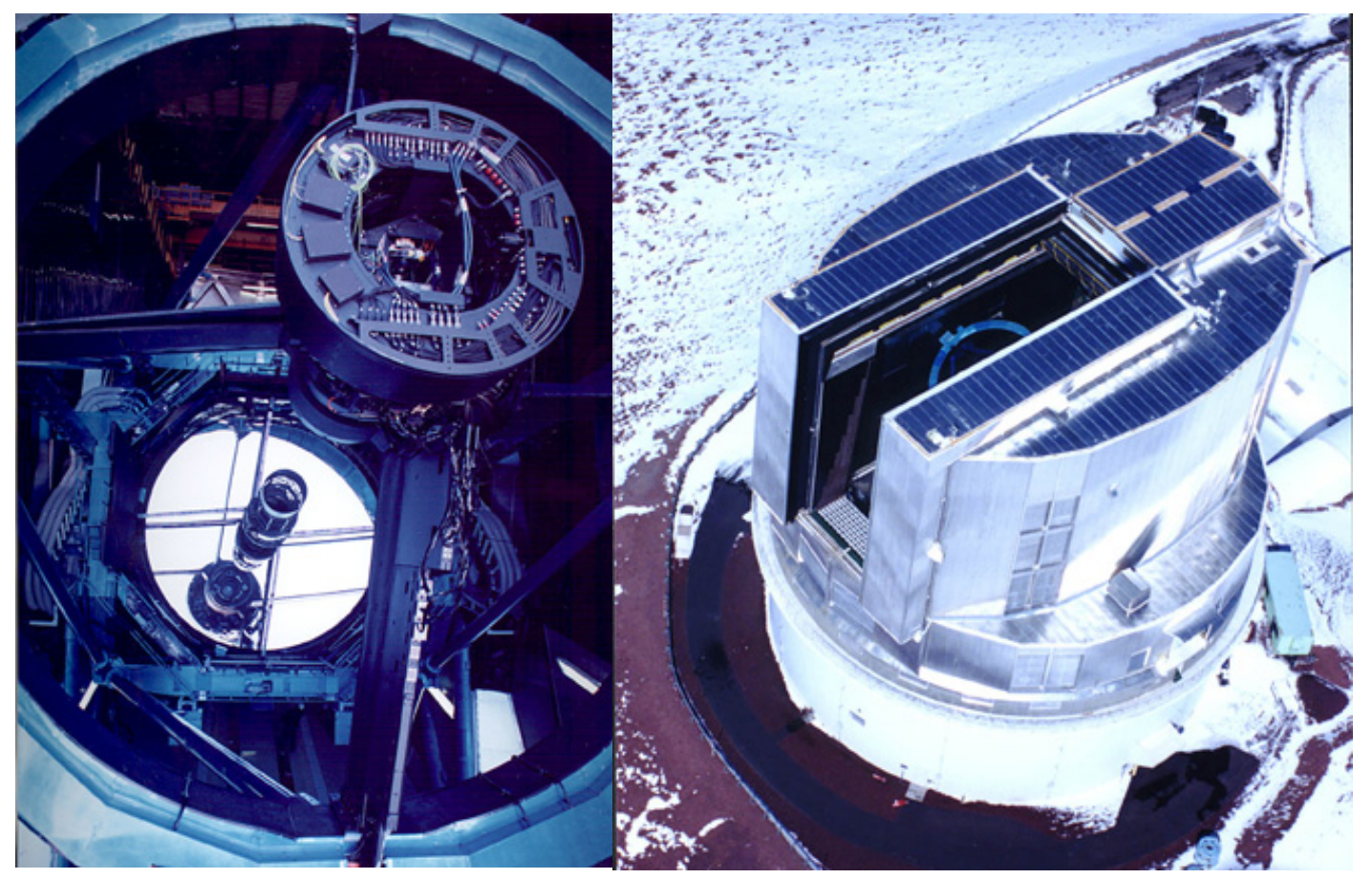

Figure 4. Subaru Telescope: Primary focus (left) and cylindrical enclosure.

\section{Basic Factors and Limitations}

Both ground-based and Space-borne astronomy of Japan made big jumps to catch up to the forefront of astronomy, based on a high historical background of astronomy, good economy and good coordination of community and industries. On the other hand we always had a serious man-power problem which should be inevitable in the rapid growth. Figure 5 shows the considerable increase of ASJ

(Astronomical Society of Japan) professional members since the late 1980's to now (roughly from 500 to 1600). Here the "professional member" includes most of the Ph-D course students. The number of female members has increased amazingly, from almost zero in 1980's to $12 \%$ of members. Such rapid increase of young astronomers is apparently a result of the jump of Japanese observational astronomy in the 1970's and 1980's. New large facilities support the following jumps. However, each increase of astronomers normally comes after the jump. Also in many countries the increase rate of position of scientists are lower than the required rate, thus we had been facing a man-power problem for many years.

Let us check the number of IAU members. The 18 Japanese IAU members in 1961 increased to 115,380 and 600 in 1970, 1994 and 2009, respectively. It seems to be a pretty big jump, but actually the percentages of Japanese members to the IAU total members are; $2.3 \%, 3.7 \%, 4.9 \%$ and $6 \%$ each, as the total IAU members has also been rapidly increasing! Japan has now 9th largest membership normalised by national population in the IAU (Netherlands and Australia are the first).

Organization of the core institute and its cooperation with the community of astronomyrelated fields (many of them are astronomers/physicists in universities) are the principal factors for the development of astronomy. Japan had developed a system for scientific research which was efficient for those fields requiring large research instruments or facilities like astronomy, particle physics, etc. Such institutes, called as Inter-University Research Institutes, are 100\% funded by the government. The $\mathrm{NAOJ}$ is one of the Inter-University Research Institutes which was reorganized from the Tokyo 
Astronomical Observatory of U-Tokyo in 1988. ISAS, Institute for Astronautical and Space Sciences, became an Inter-University Research Institutes in 1981 and also an attached institute of U-Tokyo. The merit of this type of institute is that they can act as a core institute in their fields apart from an individual university, and can develop and construct very large facilities representing the whole community.

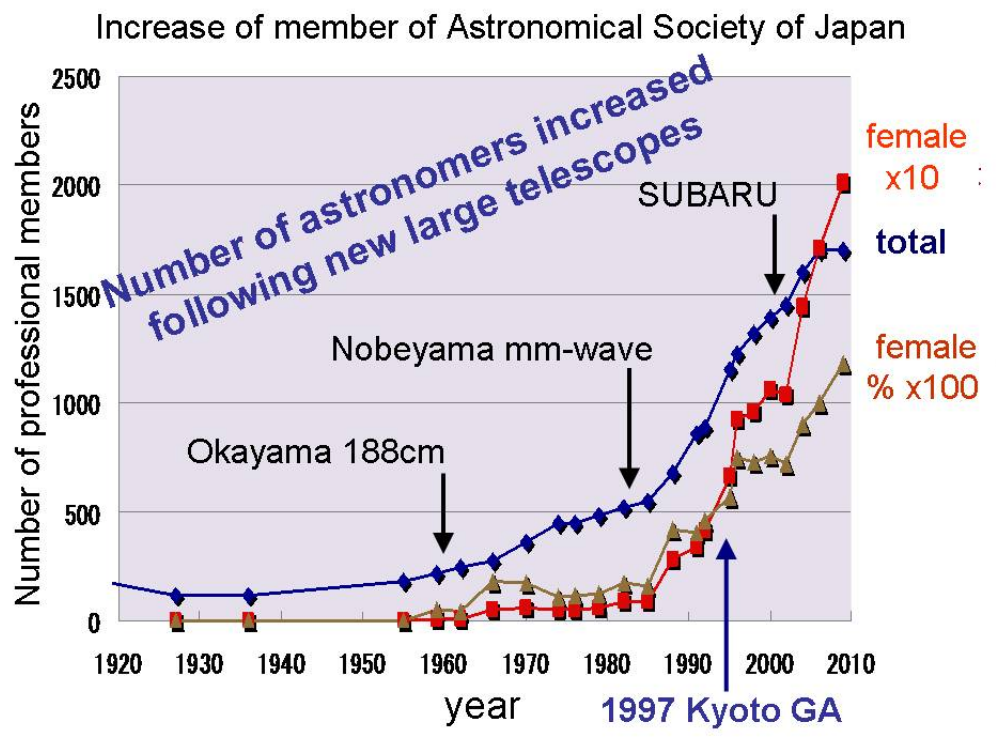

Figure 5. Professional membership of the Astronomical Society of Japan, showing a rapid increase since late 1980's.

The Nobeyama Observatory actually acted in such a role before the TAO became NAOJ. After its establishment in 1988 the NAOJ has constructed Subaru, VERA, ALMA and many other Japanese ground-based large telescopes. NAOJ, as a national core institute of astronomy, operates those telescopes, and telescope time is allocated to astronomers both in Japan and from abroad. All those processes as well as personnel committees are open to the Japanese astronomical community. The NAOJ thus supports the community but it should also be supported by community for its operation and for future developments. Therefore the cooperation and relationship between the Inter-University Research Institute and the related community is essential. Such a role of the Inter-University Research Institute differs somewhat from field to field reflecting their situation, but the ISAS (which now belongs to JAXA but still functions as such an Institute) remains in a similar situation. The NAOJ, a core national institute of ground-based astronomy, was a good system for Japan to catch-up to the top of the world astronomy, and it might also be true for many developing countries. On the other hand it is a single peak. The single peak should be high, but to be high it needs a wide skirt, i.e. a good size and active astronomical community.

In addition, both core institutes and universities need good public understanding. Fortunately, Japanese astronomers enjoy a high public interest in astronomy; Japan has 200 open observatories operated by local governments to show the universe to children and public, and 300 planetaria (second highest in the world after the USA), and the outreach activities of Japanese amateur astronomers are among the highest in the world. We have also tried many years to improve our contact with the public. More than 200 million people have visited Nobayama Radio Observatory, and the hit rate of the NAOJ website exceeds 50 million/year. We are proud that NAOJ is the best-known scientific institute in Japan.

\section{Conclusion: Accelerate the Coordination and Cooperation}

Astronomy is now a Big Science in Japan, but inevitably many limitations exist in such rapid developments. As we have already discussed, Japan has only a single core institute, NAOJ, and powering astronomy in universities is always important. Manpower is still difficult, and education is becoming more and more important. The budget is always a difficult matter everywhere, especially in the recent economical situation. We might have to cool the very high acceleration rate towards the next large project considering the realistic timescale in the coming era of global cooperation. 
International cooperation should be the most important direction in the future. On the other hand, I believe that the regional cooperation is still essentially important for long-term future of astronomy. In Asia, the East Asia VLBI Network consortium and East Asian ALMA Research Center have mostly established cooperation, and more regional cooperation plans are being discussed for the future. The four core observatories of China-Beijing (NAC), China-Taipei (ASIAA), Japan (NAOJ) and Korea (KASI) have established the EACOA (East Asian Core Observatories Association) in September 2005 after 20 years efforts of astronomer-base cooperation of EAMA (East Asian Astronomers Meeting). The SEAAN (South East Asian Astronomers Network) was also established recently by Indonesia, Laos, Malaysia, Philippines, and Thailand. Such new movements based on improvements of economy and science in those regions, and will open up new possibilities of "acceleration of the rate of discoveries" with numbers of high peaks and a very broad outskirt.

\section{References}

[1] M. Morimoto and N. Kaifu, A Search for Interstellar Para-Formaldehyde, 1972, Mem.Soc.Roy.Sci., Liege, 6, Serrie 3, 467.

[2] N. Kaifu, Nobeyama 45-m Telescope, 1984, in the URUSI International Symposium, "Milimeter and Submillimeter Wave Radio Astronomy", Granada, Spain, pp.5-10.

[3] N. Kaifu et.al., The First Light of the Subaru Telescope: A New Infrared Image of the Orion Nebula, 2000, Publ. Astron. Soc. Japan, 52, 1-8.

[4] N. Kaifu, Ground-Based Astronomy in Japan, 1996, in proceedings of Third East-Asian Meeting on Astronomy, "Ground-Based Astronomy in Asia", ed. N.Kaifu, National Astronomical Observastory of Japan.

[5] http://www.narit.or.th/seaan/ 